\title{
Modelling the Bivariate Dependence Structure of Exchange Rates Before and After the Introduction of the Euro: a Semi-Parametric Approach
}

March 2009

\author{
Gianna Boero $^{1} \quad$ Param Silvapulle $^{2} \quad$ Ainura Tursunalieva $^{3}$
}

\begin{abstract}
This paper investigates the bivariate dependence structure for three pairs of exchange rates measured against the US dollar: Euro and Japanese yen, Euro and British pound, Euro and Swiss franc, over the period January 1994 to November 2007. The Deutsche mark (DM) is used for the pre-euro period. By using non-parametric plots and copula models estimated by semi-parametric methods, we are able to detect changes in the dependence structure from the pre-euro to the post-euro period for the pairs DM (Euro)Japanese yen, and DM (Euro)-British pound, with major changes occurring during the initial years of the launch of the new currency. For these two pairs of exchange rates we also capture asymmetric tail dependence, implying different degrees of comovements during appreciations and depreciations against the dollar. The dependence between the DM (Euro) and the Swiss franc remains unchanged, both in strength and structure, over the whole sample period, reflecting a marked tendency of the Swiss franc to follow the fluctuations of the DM and Euro against the dollar. The results may be of interest for international trade, international portfolio diversification and currency risk management.
\end{abstract}

Keywords: Copulas, non-parametric plots, semi-parametric methods, simulation, tail dependence.

JEL Classification: C14, G15, F31

${ }^{1}$ Corresponding author: Department of Economics, University of Warwick, Coventry, CV4 7AL, UK, Email: gianna.boero@warwick.ac.uk Tel: +44 24 76523796, fax: +44 24 76523032 .

${ }^{2}$ Department of Econometrics and Business Statistics, Monash University, Caulfield East, VIC 3145, Australia

${ }^{3}$ Department of Econometrics and Business Statistics, Monash University, Caulfield East, VIC 3145, Australia 


\section{Introduction}

Movements of financial markets can be extremely complex and highly volatile, and are largely driven by continuous global trading activities of major financial institutions and investors. The global currency markets are known to dominate other markets such as stock and bond markets. The average daily turnover of the foreign exchange, or forex, market has been reported to be over US \$3 trillion in 2007 (Bank for International Settlements, Triennial Central Bank Survey, 2007), approximately eight times the total size of all major equity markets. The increased importance of exchange rates, as observed by Copeland (2008), is the result of "the internationalization of modern business, the continuing growth in world trade relative to national economies, the trend towards economic integration (in Europe, at least), and the rapid pace of change in the technology of money transfer" (Copeland, page 1). Continuing with Copeland, "whatever the subject under discussion - the outlook for the domestic or world economy, stock markets, industrial competitiveness, even the outcome of the next election - the answer almost invariably turns out to revolve around the exchange rates". Because of its connections to all other financial markets, fluctuations in the foreign exchange market have pervasive effects and are influenced by the behaviour of the many players involved - governments, central banks, large banks, multinational corporations, currency speculators. This study wants to contribute to the understanding of movements and comovements of exchange rates, with an application to some of the most traded currencies in the forex market: the US dollar, the Euro, the Japanese yen, the British pound and the Swiss franc.

More specifically, the primary objective of this paper is to investigate the nature of the underlying dependence structure between pairs of bilateral exchange rates measured against the US dollar - precisely, the Euro (formerly the Deutsche mark) and the Japanese yen (JY), the Euro and the British pound (BP), the Euro and the Swiss franc (SF). The study, based on the theoretical framework of conditional copulas (Patton, 2006), is conducted before and after the introduction of the Euro in order to assess whether the launch of the new currency - and the uncertainty associated with the initial 
transition period - has had an impact on the nature of dependence between the exchange rates.

An issue that has received considerable attention in recent years is measuring the dependence in international financial markets during extreme fluctuations. For example, stock returns appear to become more related when they are large and negative (lower tail dependence) than when they are large and positive (upper tail dependence), a phenomenon which is known as financial contagion and which cannot be captured by simple correlation (see, for example, Ang and Chen, 2002, Login and Solnik, 2001, Granger and Silvapulle, 2001 and references therein). Copulas are emerging as attractive models for capturing and measuring various forms of dependence, and have found useful applications, especially for the analysis of the behaviour of returns on financial assets (a partial list of recent contributions includes Bouyé and Salmon, 2002, Ang and Bekaert, 2002, Jondeau and Rockinger, 2006, Campbell et al., 2008, Sun et al., 2008).

The use of copulas to study exchange rate dependence is more recent, and still very limited (see Patton 2006, Hurd, Salmon and Schleicher, 2005, Dias and Embrechts, 2007). Several suggestions on possible sources of asymmetric exchange rate dependence have been proposed in previous studies. As reported in Patton (2006), asymmetric dependence between exchange rates may reflect asymmetric responses of central banks to exchange rate movements. More precisely, it is suggested that possible asymmetries may arise depending on the relative weight put by central banks on two main objectives: maintaining competitiveness and preserving price stability. A preference for price stability would induce a central bank to intervene in order to match the appreciation of other currencies towards the US dollar, for example. On the other hand, a preference to defend competitiveness would induce central bank intervention to match depreciation when other currencies depreciate against the dollar. Thus exchange rates may show stronger dependence during periods of large depreciations or appreciations than during normal periods. Asymmetric dependence between exchange rates may also be generated by currency portfolio rebalancing activities involving shifting funds from other currencies to the dollar when the latter is strong, and from the dollar to the next most important currencies when the dollar is weak, therefore resulting in greater dependence between 
currencies in the first instance than in the second. These phenomena and the risk associated with exchange rate fluctuations have economic and financial implications for international trade, international portfolio diversifications, and can have huge effects on "carry trade", a strategy in which investors borrow funds in the currency of a country that has low interest rates (such as Switzerland and Japan) and invest these funds in a country with high interest rates. See also Sarno and Taylor (2002) for a comprehensive survey of the theoretical and empirical literature on foreign exchange market intervention.

In this paper we use non-parametric plots and copula models estimated by a semiparametric method to assess the presence of dependence and tail dependence between the three pairs of exchange rates described above, over the period 2 January 1994-2 November 2007. According to Sklar (1959) theorem, any $n$-dimensional joint distribution function may be decomposed into $n$ marginal distributions and a copula, a function which completely characterises the dependence among the $n$ variables (see Joe, 1997 and Nelsen, 2006 for a detailed treatment of copulas). The standard Pearson correlation cannot capture complex dependence structures, as it is only a symmetric, linear dependence metric. The attraction of the copula method comes from its flexibility, enabling representation of the joint distribution and dependence structure between random variables even when their marginal distributions are non-elliptic and each margin has a different distribution, and from its ability to separate the measure of dependence from the marginal distributions. Another attractive feature of this approach is its invariance property by which the copula and the association parameter are invariant under continuous and monotonically increasing transformation of the marginal variables. This means that the same copula may be used for the joint distribution of exchange rates and of exchange rate returns. Estimating copula models typically involves two stages: first the estimation of the margins, then the estimation of the copula, to obtain a measure of the dependence between the margins. In practice, neither the underlying margins nor the copulas (multivariate distributions) are known to researchers and they can be unwittingly misspecified.

One of the distinctive features of our paper is the use of a semi-parametric approach for modelling copulas, whereby the marginal distributions are assumed to be unknown and 
estimated by a non-parametric method, while Maximum Likelihood Estimation (MLE) is used for the copulas. Our approach is based on an extension of Genest et al. (1995) and is inspired by recent findings by Kim et al. (2007a, b, and 2008) who show that the semiparametric estimation method for modelling copulas is robust when the margins are misspecified, and establish its superiority over fully parametric counterparts, such as Inference Function and MLE methods.

Briefly stated, our results can be summarised as follows. For the first two pairs of exchange rates, DM (Euro)-Japanese yen and DM (Euro)-British pound, we are able to detect changes in the dependence structure over three distinct periods - pre-euro, transition and post-euro period - where the transition is defined as the period during which the national currencies were still used in parallel with the new currency for domestic transactions. For these two pairs of exchange rates we also capture changes in tail dependence, implying different degrees of comovements during appreciations and depreciations against the dollar. For the third pair of exchange rates considered in this study, the DM (Euro) and the Swiss franc, we find that the conditional joint distribution and the implied dependence remains unchanged, both in strength and structure, across the three subsamples, reflecting a marked tendency of the Swiss franc to follow the fluctuations of the DM and of the Euro against the dollar.

This paper is planned as follows. Section 2 introduces the methodology used in our analysis, namely, non-parametric graphical methods - chi and K-plots - based on concordance metrics that are closely related to copulas, standard copula models that have recently become very popular in empirical studies, and a semi-parametric estimation method for copulas. Section 3 describes the exchange rate data series and their properties. Section 4 compares the chi- and K-plots of simulated data with those of the observed exchange rate returns series and reports the results of the estimated bivariate copulas for the three pairs of exchange rate returns. Section 5 discusses the implications of the findings and concludes. 


\section{Methodological issues}

In this section, we outline the non-parametric plots employed to assess the significance of dependence and tail dependence; some standard copula functions that have been widely used in recent studies in empirical finance, and a semi-parametric approach for copula modelling.

\subsection{Nonparametric plots for assessing dependence and selecting suitable copulas}

The chi-plot and K-plot are rank-based graphical methods which can be used in conjunction with a scatterplot of the raw data to detect possible association between two variables. Both procedures share the key property of invariance with respect to monotone transformations of the marginal distributions. In what follows we will briefly discuss the concepts used in the chi-plot and K-plot and outline the hypotheses and test statistics used in these plots.

\subsubsection{Chi-Plot}

The chi-plot, developed by Fisher and Switzer (1985), is based on the transformation of $n$ pairs $\left(X_{i}, Y_{i}\right)$ into $n$ pairs $\left(\lambda_{n_{i}}, \chi_{n_{i}}\right)$, with the intention of revealing, through a scatterplot of the transformed pairs, more detailed information on the nature of dependence between the raw series. Intuitively, $\chi_{n_{i}}$ provides an indication of the association, separately for each data point $\left(X_{i}, Y_{i}\right)$, and $\lambda_{n_{i}}$ measures the distance of each pair $\left(X_{i}, Y_{i}\right)$ from the centre of the data set (the bivariate median). The chi-plot is approximately horizontal under the null hypothesis of independence, while deviations from the horizontal line $\chi=0$ (with allowance for sampling variability) indicate departures from independence. The graphical aspect of the chi-plot depends on the form of the $\left(X_{i}, Y_{i}\right)$ association (as well as on the choice of the data transform $\lambda_{n_{i}}$ ), and may be useful in the identification of the copula underlying the data (see Genest and Boies, 2003).

To describe this method, let $\left(X_{1}, Y_{1}\right), \cdots,\left(X_{n}, Y_{n}\right)$ of size $n \geq 2$ be a random sample from a continuous bivariate cumulative distribution $H$. Further, let $F$ and $G$ be the marginal 
distributions of $X$ and $Y$, respectively. For each data point $\left(X_{i}, Y_{i}\right)$, define:

$$
\begin{aligned}
& H_{i}=H\left(X_{i}, Y_{i}\right)=\sum_{j \neq i} I\left(X_{j} \leq X_{i}, Y_{j} \leq Y_{i}\right) /(n-1), \\
& F_{i}=F\left(X_{i}\right)=\sum_{j \neq i} I\left(X_{j} \leq X_{i}\right) /(n-1),
\end{aligned}
$$

and

$$
G_{i}=G\left(Y_{i}\right)=\sum_{j \neq i} I\left(Y_{j} \leq Y_{i}\right) /(n-1),
$$

where the indicator function $I(E)$ is defined as 1 if the event $E$ occurs and 0 otherwise. If $X$ and $Y$ are independent, then it is expected that $H_{i}=F_{i} G_{i}$, except for sampling variability, at each of the sample cut-points. Under the null hypothesis of independence, the standardized test statistic

$$
\chi_{n_{i}}=\frac{H_{i}-F_{i} G_{i}}{\sqrt{F_{i}\left(1-F_{i}\right) G_{i}\left(1-G_{i}\right)}},
$$

for $i=1,2, \cdots, n$, asymptotically follows a normal distribution with mean zero and variance $1 / n$, for a given cut-point $\left(X_{i}, Y_{i}\right)$. The statistic $\chi_{n_{i}}$ is clearly a correlation coefficient between dichotomized $X$ and $Y$, and therefore, all values of $\chi_{n_{i}}$ are expected to lie in the interval $[-1,1]$.

Further, define

$$
\lambda_{n_{i}}=4 S_{i} \max \left\{\left(F_{i}-1 / 2\right)^{2},\left(G_{i}-1 / 2\right)^{2}\right\}
$$

where

$$
S_{i}=\operatorname{sign}\left\{\left(F_{i}-1 / 2\right)\left(G_{i}-1 / 2\right)\right\}
$$

$\lambda_{n_{i}} \in[-1,1]$ measures the distance of the data point $\left(X_{i}, Y_{i}\right)$ from the centre of the dataset, showing clustering when $X$ and $Y$ are associated. Specifically, $\lambda_{n_{i}}$ will be positive if $X$ and $Y$ are positively correlated, and negative if $X$ and $Y$ are negatively correlated. Details on the properties of chi-plots and examples of applications to artificial and real data can be found in Genest and Boies (2003) and Fisher and Switzer (1985, 2001).

\subsubsection{Kendall-Plot}

Genest and Boies (2003) proposed an alternative rank-based procedure, known as the Kplot, which adopts the familiar concept of probability plot (the Q-Q plot) to the detection 
of dependence. A lack of linearity of the standard Q-Q plot is an indication of nonnormality of the distribution of a random variable. Similarly, in the absence of association, the K-plot is close to a straight line, while the amount of curvature in the plot is characteristic of the degree of dependence in the data, and is related in a definite way, to the underlying copula. This method is closely related to the Kendall's $\tau$-statistic (Kendall 1938), from which it takes the name.

To define the K-plot, consider the probability integral transformation of the component $\left(X_{i}, Y_{i}\right)$ to $\left(U_{i}, V_{i}\right)=\left(F\left(X_{i}\right), G\left(Y_{i}\right)\right)$, for $i=1, \cdots, n$, so that $U_{i}$ and $V_{i}$ are uniform $(0,1)$. Typically, the marginal distributions $F(X)$ and $G(Y)$ are unknown, so plots of the pairs of the empirical distributions $\left(\hat{F}_{n}\left(X_{i}\right), \hat{G}_{n}\left(Y_{i}\right)\right)$, for $1 \leq i \leq n$, or equivalently, of the pairs of the ranks of $X_{i}$ and $Y_{i}$, say $\left(R_{i} / n, S_{i} / n\right)$, can be used. Following Genest and Boies (2003), because monotone transformations of the marginal distributions yield invariant ranks, the transformed data may be regarded as observations from the unique underlying copula

$$
C(u, v)=H\left\{F^{-1}(u), G^{-1}(v)\right\}, \text { for } 0 \leq u, v \leq 1,
$$

associated with $H$, defined above. The construction of a K-plot requires, first of all, to order $H_{i}$, as $H_{(1)} \leq \cdots, \leq H_{(n)}$, then to plot the pair $\left(W_{(i: n)}, H_{(i)}\right)$, for $i=1, \cdots, n$, where $W_{(i: n)}$ is the expectation of the $i$ th order statistic in a random sample of size $n$, drawn from the distribution $K_{0}$ of the $H_{i}$, under the null of independence. The form of the bivariate distribution $K_{0}$ (when $H=F G$ ) is given as follows:

$$
K(\omega)=K_{0}(\omega)=\operatorname{Pr}\{U V \leq \omega\}=\omega-\omega \log (\omega),
$$

for $0 \leq \omega \leq 1$, where $U$ and $V$ are independent uniform random variables on the interval $[0,1]$. This choice of $K_{0}(\omega)$ is then used to compute the $W_{(i: n)}$ required for the plot. The expression for $W_{(i: n)}$ is given in Genest and Boies (2003, equation 3). 


\subsection{Copula specifications}

The chi-plot and Kendall-plot introduced above can reveal complicated dependence structures and are directly related to the concept of copula. Copulas are flexible tools for constructing joint distributions and provide information about dependence. To model the conditional dependence between pairs of exchange rates, we employ various copula functions that are frequently applied in finance, offering different dependence structures. These include elliptic copulas (Normal and Student $t$ ), Archimedean copulas (Clayton and Gumbel) and the symmetrised Joe-Clayton copula. The elliptic copulas impose a symmetric dependence structure, while the other specifications allow for some degree of asymmetric dependence in either direction, that is, joint negative events (lower left quadrant) are allowed to be more or less dependent than joint positive events (upper right quadrant). Below we give a brief description of these copula functions, while more details can be found in Joe (1997) and Nelsen (2006), among others. See also Patton (2007) for an excellent review of the literature on copula models for financial time series.

\subsubsection{Elliptic Copulas}

The Gaussian or Normal copula is defined as:

$$
C(u, v)=\Phi_{\rho_{X Y}}\left(\Phi^{-1}(u), \Phi^{-1}(v)\right)
$$

where $\Phi_{\rho X Y}$ is the standard bivariate normal distribution with correlation coefficient $\rho_{X Y}$ and $\Phi^{-1}$ is the inverse of the univariate standard normal distribution function (cdf). The normal copula distribution is expressed as follows:

$$
\Phi_{\rho_{X Y}}\left(\Phi^{-1}(u), \Phi^{-1}(v)\right)=\int_{-\infty}^{\Phi^{-1}(u)} \int_{-\infty}^{\Phi^{-1}(v)} \frac{1}{2 \pi \sqrt{1-\rho_{X Y}^{2}}} \exp \left\{\frac{-\left(s^{2}+t^{2}-2 \rho_{X Y} s t\right)}{2\left(1-\rho_{X Y}^{2}\right)}\right\} d s d t
$$

where $\rho_{X Y}$ is the copula parameter $\left(-1<\rho_{X Y}<1\right)$.

The Normal copula allows for equal degrees of positive and negative dependence and has the property of asymptotic independence, that is extreme events occur independently in each margin, no matter how high is $\rho_{X Y}$.

The Student copula is extracted from the bivariate Student $t$ distribution, and takes the 
form

$T_{\rho, v}(u, v)=t_{\rho, v}\left(t_{v}^{-1}(u), t_{v}^{-1}(v)\right)=\int_{-\infty}^{t_{v}^{-1}(u)} \int_{-\infty}^{t_{v}^{-1}(v)} \frac{1}{2 \pi \sqrt{1-\rho^{2}}}\left\{1+\frac{\left(s^{2}+t^{2}-2 \rho s t\right)}{v\left(1-\rho^{2}\right)}\right\}^{-\frac{v+2}{2}} d s d t$

where $t_{v}^{-1}$ denotes the quantile function (inverse of the cdf) of the standard univariate $t$ distribution with $v$ degrees of freedom, and $\rho$ is the correlation coefficient.

The Student copula has received much recent attention for modelling financial returns, for its ability to capture (better than the Gaussian copula) the dependence of extreme values, a phenomenon typically observed in financial return data. Specifically, the Student $t$ copula displays symmetric tail dependence and asymptotic upper tail dependence, even for zero or negative correlation, with dependence rising as $v$ decreases and the margins become more heavy-tailed (see Embrechts et al. 2002).

For all elliptical copulas the relationship between the Kendall's tau $\tau$ and the copula parameter $\rho_{X Y}$ is given by $\rho_{X Y}=\sin \left(\frac{\pi}{2} \tau\right)$.

\subsubsection{Some Archimedean copulas}

Archimedean copulas have a variety of dependence structures. These copulas may be expressed in the following form:

$$
C(u, v)=\phi^{-1}[\phi(u)+\phi(v)]
$$

where $\phi$ is a generator function known as Archimedean generator for $\phi: I \rightarrow \mathfrak{R}^{+}$that is continuous, decreasing, convex and satisfies $\phi(1)=0$. We consider two copulas with asymptotic tail dependence: Clayton (1978) and Gumbel (1960) and their respective survival copulas.

The Clayton copula is defined as:

$$
C(u, v)=\max \left[\left(u^{-\theta}+v^{-\theta}-1\right)^{-\frac{1}{\theta}}, 0\right]
$$

where the parameter $\theta$ has range $[(-1,0) \cup(0, \infty)]$. 
The Gumbel copula is defined as:

$$
C(u, v)=\exp \left\{-\left[(-\ln u)^{\theta}+(-\ln v)^{\theta}\right]^{\frac{1}{\theta}}\right\}
$$

where $\theta \in[1, \infty]$. The Clayton copula captures lower tail dependence, and the Gumbel copula captures upper tail dependence.

Survival Clayton and survival Gumbel copulas are associated with bivariate uniform pairs $(1-u, 1-v)$ and the left and right tails are exchanged, so the Survival Gumbel copula allows for lower tail dependence, while the Survival Clayton allows for upper tail dependence.

\subsubsection{The symmetrised Joe-Clayton copula}

The symmetrised Joe-Clayton copula (SJC) was proposed by Patton (2006) as a modification of the "BB7" copula of Joe (1997), defined as:

$$
C_{J C}\left(u, v \mid \tau^{U}, \tau^{L}\right)=1-\left(1-\left\{\left[1-(1-u)^{k}\right]^{-\gamma}+\left[1-(1-v)^{k}\right]^{-\gamma}-1\right\}^{-\frac{1}{\gamma}}\right)^{\frac{1}{k}}
$$

where $k=\frac{1}{\log _{2}\left(2-\tau^{U}\right)}, \gamma=\frac{-1}{\log _{2}\left(\tau^{L}\right)}$, and $\tau^{U} \in(0,1), \tau^{L} \in(0,1)$ are two parameters which measure the upper and lower tail dependence, respectively. The modification proposed by Patton given by:

$$
C_{S J C}\left(u, v \mid \tau^{U}, \tau^{L}\right)=0.5 \cdot\left(C_{J C}\left(u, v \mid \tau^{U}, \tau^{L}\right)+C_{J C}\left(1-u, 1-v \mid \tau^{U}, \tau^{L}\right)+u+v-1\right)
$$

nests symmetry $\left(\tau^{U}=\tau^{L}\right)$ as a special case (see Patton, 2006, for details).

\subsection{Semi-parametric estimation of copulas}

Various methods have been followed to estimate copulas. The most direct estimation method is simultaneous estimation of all parameters using the full maximum likelihood approach (FML). A second method follows a sequential two-step maximum likelihood approach (TSML), in which the marginals are estimated in the first step, and the dependence parameter is estimated in the second step using the copula. This second method has additional variants according to whether both steps are implemented parametrically (either with ML or Inference Function methods), non-parametrically, or semi-parametrically. Moreover, there are variants of this two-step approach depending on 
the method used to estimate the variance of the dependence parameter in the second stage.

In this paper we adopt a two-step method which enables us to exploit the attractive feature of copulas for which the dependence structure is independent of the marginals. This method, described in detail below, is a natural extension of the method proposed by Genest et al. (1995) for independent and identically distributed observations. Specifically, we implement a semi-parametric (SP) method in which a non-parametric method is used to estimate the marginal distributions in the first stage, while a parametric method is used to estimate the copula in the second stage. Since our interest is in conducting statistical inference on copula and tail dependence parameters, the use of parametric copulas would permit this (see Kim et al. 2007a).

Copulas were originally developed for margins with i.i.d. observations. It is well known that time series, in particular high frequency series, are serially correlated and highly volatile, following ARCH/GARCH-type processes. In such cases, however, it has been shown that margins for i.i.d. can be replaced with those for ARMA-ARCH/GARCH filtered residuals (see, Kim et al., 2007b, 2008, and the references therein). In our analysis, we fit univariate ARMA-GARCH models on the individual exchange rate returns, assuming normality. We then estimate empirical distributions on the $n$ filtered returns, say $X=\left(x_{1}, \ldots, x_{n}\right)$ and $Y=\left(y_{1}, \ldots, y_{n}\right)$, represented by the residuals from these univariate models. Assuming that $X$ and $Y$ are independent and identically distributed variables with continuous marginal distribution functions, we estimate sample empirical distributions as:

$$
\begin{aligned}
& \hat{F}_{X}(x)=\frac{1}{n+1} \sum_{i=1}^{n} I_{\left\{X_{i}<x\right\}} \\
& \hat{F}_{Y}(y)=\frac{1}{n+1} \sum_{i=1}^{n} I_{\left\{Y_{i}<y\right\}}
\end{aligned}
$$

where $I_{\{.\}}$is an indicator function, and $\hat{F}_{X}(x)$ and $\hat{F}_{Y}(y)$ are pseudo-observations approximately uniformly distributed in $[0,1]$. We then estimate by MLE methods copula models of the type

$$
\hat{F}_{X Y}(x, y ; \theta)=C\left(\hat{F}_{X}(x), \hat{F}_{Y}(y) ; \theta\right)
$$


where $\theta$ represents a set of parameters measuring dependence for the bivariate set of pseudo observations $\left(\hat{F}_{X}(x), \hat{F}_{Y}(y)\right.$. This second step involves estimating $\theta$ by the maximiser of the pseudo log-likelihood function

$$
\sum_{i=1}^{n} \log c\left(\hat{F}_{X}\left(x_{i}\right), \hat{F}_{Y}\left(y_{i}\right) ; \theta\right)
$$

where $c(\cdot ; \theta)$ stands for the density of the parametric copula family. We consider several copulas, with one or two parameters, capturing symmetric and possible asymmetric dependence, and select a suitable copula based on the Akaike Information Criterion and the goodness of fit test proposed by Breymann et al. (2003). From the selected copula we then obtain estimates of the corresponding lower and upper tail dependence parameters for the joint distribution of the exchange rate pairs. Standard errors are estimated using the estimator given in Genest et al. (1995, section 3). Fully parametric estimation methods such as Inference Function (IF) and MLE have been proved successful for copula estimation in several studies (see, for example, Patton, 2006 and Rodriguez, 2007). However, in practice, the true margins are largely unknown to researchers, so testing for marginal distribution model misspecification is critical in fully parametric copula estimation, as misspecification of the margins would result in a misspecified copula model. One of the attractive properties of the semi parametric method implemented in our study is that the time series structure ARMAARCH/GARCH can be estimated simply assuming normal distribution. Each marginal distribution is estimated nonparametrically by the empirical distribution function, thus allowing the distribution of the marginals to be not restricted by parametric families. Kim et al. (2007a, 2008) have shown that the semi parametric method implemented in our study is robust when the margins are misspecified and as efficient as MLE in moderate sample sizes (greater than 100). As financial markets provide high-frequency data with large sample sizes, semi-parametric methods provide an attractive approach to investigate the dependence structure in the exchange rate returns. 


\section{The exchange rate data series}

In this study we use daily data for four currencies: Euro (DM before 1999), British Pound, Swiss Franc and Japanese Yen. All currencies are measured against the U.S. dollar and cover the period January 2, 1994 to November 2, 2007. The data were collected from DATASTREAM and transformed into daily logarithmic returns. In order to explore whether the introduction of the Euro has affected the relationship between exchange rates, we examine the dependence in the pairs DM(Euro)-BP, DM(Euro)-SF and DM(Euro)-JY, in three sample periods: (i) January 1994 - December 1998; (ii) January 1, 1999 - February 28, 2002; and (iii) March 1, 2002 to November 2, 2007. We refer to these sub samples, respectively, as the pre-euro period, the transition period (during which the national currencies were still used for domestic transactions), and the post euro period (disappearance of the national currencies).

The time series plots of each pair of exchange rates are given in Figure 1, while Table 1 reports the summary statistics of the exchange rate returns for the three sample periods. The DM(Euro)/USD shows an upward trend (depreciation) during the transition period, followed by a downward trend thereafter (a tendency towards appreciation). Similar patterns - a tendency towards depreciation in the transition period and appreciation in the post euro period - are displayed by the BP/USD, while the movements exhibited by the $\mathrm{BP} / \mathrm{USD}$ and the DM/USD in the pre-euro period are less related, as also documented by the Pearson's correlation coefficient reported in Table 1. The fluctuations of the $\mathrm{DM}$ (Euro) are mimicked very closely by the Swiss franc over the whole period, and this similarity is also reflected in the summary statistics for the marginal distributions of the exchange rate returns across subperiods. The JY/USD and the DM(Euro)/USD exchange rates show some close degree of comovement in the pre-euro and post-euro periods, while they appear to be more unrelated during the transition period. From Table 1 we notice that the coefficient of correlation is 0.536 and 0.528 in the pre and post euro periods, respectively, and only 0.118 in the transition period. With respect to other sample statistics - skewness and kurtosis - it is clear that the marginal distributions are far from normal, as also indicated by the Jarque-Bera test. Moreover, the table indicates changes in sample statistics across subperiods, more visible for some currencies than 
others. For example, the JY/USD shows the highest kurtosis (9.770) and negative asymmetry $(-0.841)$ in the pre-euro period, with both measures much attenuated in the transition and post-euro periods, though still indicating departure from a normal distribution. The second highest kurtosis (0.938) is shown by the BP/USD exchange rate returns in the pre-euro period, while in the transition and post-euro periods the value of the kurtosis coefficient is much closer to 3 , indicating tails of the marginal distribution not too far away from normal.

Table 1 also reports the estimates of the Kendall's $\tau$ on the bivariate relationship between the DM(Euro)/USD and the other three exchange rate returns. Clearly, the Pearson's correlation coefficient $\rho$ is consistently higher than $\tau$. The former measures the linear dependence and is not robust to outliers, while the latter measures any form (even complex) of association between two variables. Therefore, we focus on the Kendall's $\tau$ to infer the nature of the relationships among these currencies. The estimated $\tau$ indicates that the dependence between the DM(Euro)/USD and JY/USD is approximately the same in both the pre-euro and post-euro periods, but close to zero in the transition period, as already pointed out in discussing the Pearson coefficient above. The dependence between the DM(Euro)/USD and SF/USD, on the other hand, is very strong across all three sample periods. The dependence between the DM(Euro)/USD and $\mathrm{BP} / \mathrm{USD}$ has almost doubled moving from the pre-euro to the post-euro period. In what follows, we investigate these relationships by modelling copulas and estimating the corresponding dependence and tail dependence parameters.

\section{Empirical results}

In this section we construct chi- and K-plots for the data generated from the copulas outlined in section 2.2, and for the bivariate exchange rate returns (Euro, JY), (Euro, SF) and (Euro, BP) for three sample periods (pre-euro, transition, post-euro). We use semiparametric estimation methods for copula modelling and information criteria and goodness-of-fit tests for selecting suitable copulas. 


\subsection{Results of Chi-plots and K-plots}

\subsubsection{Plots for simulated data}

To identify suitable copulas that capture the underlying tail dependence in the exchange rates, first, we adopt a graphical device and study the shapes of chi- and K-plots produced from simulated draws from the copulas specified in section 2.2. Then we compare the plots of the simulated data with those of the exchange rate returns. In simulating data from copulas, we set the values for Kendall's $\tau$ and sample sizes to be the same as those reported in Table 1 for the exchange rates series over the three sample periods. Given these Kendall's $\tau$ values, the corresponding copula parameters can be computed. By inspection, we choose copulas that produce plots which closely match with those obtained from the exchange rate series. The results for the bivariate normal copula are used as benchmarks. The plots are exhibited in Figures 2 and 3.

From section 2.1, recall that under the null hypothesis of independence, the points on the chi-plots are expected to be randomly scattered around the line $\chi_{T_{i}}=0$. Given the large samples studied in this paper, the 95 per cent confidence intervals are expected to be very close to $\chi_{T_{i}}=0$. By construction, large positive and large negative deviations of the two generated series from the median should lie on the right hand side of the plot, while deviations with opposite signs lie on the left hand side. Further, the peak of the chi-plot corresponds to the Kendall's $\tau$ value. Since the lower and upper tail dependence arises due to large negative deviations and large positive deviations, respectively, how the observations are scattered around the far-end of the right hand side of these plots is of considerable interest. The shape of the plot will be used to determine the presence of lower and upper tail dependence. The chi-plot for the normal copula shows that observations are closely clustered within the bell-shaped curve which converges to the (zero) horizontal line on both sides of the plot. The K-plot spans from zero to the very top end and almost touches the diagonal line, which is an indication of the absence of tail dependence for the normal copula.

The chi-plot for the Student t copula has observations clustered in a wider area on the right hand side of the plot, compared with that for the normal copula, and the plot does not converge to zero. These results indicate the presence of both upper and lower tail 
dependence. The distinct difference of the K-plot for the Student t copula is that the line at the very top right and bottom left corners does not converge to the diagonal line, confirming the presence of upper and lower tail dependence.

The chi-plot for the Clayton copula exhibits an interesting pattern following two separate directions on the right hand side of the plot: one branch of the plot converges to the horizontal line, while the other moves upwards, with this separation being more prominent when $\tau$ is as large as 0.8 . The K-plot exhibits a curve that is clearly further away from the diagonal line in the lower part of the plot, whereas at the top end of the plot the curve touches the diagonal line, indicating absence of upper tail dependence. The chi-plot for the Gumbel copula, on the other hand, exhibits observations which do not converge to the horizontal line on the right hand side of the plot. The K-plot clearly shows that at the origin, the curve touches the diagonal line, indicating absence of lower tail dependence, while the upper part of the curve does not touch the diagonal line, indicating upper tail dependence.

The chi-plot for the SJC copula shows that observations are not distributed along the rotated bell-shaped area on the right hand side of the plot, which does not converge to zero. Further, the K-plot does not converge to the diagonal line, indicating presence of lower and upper tail dependence. Further, contrary to the $t$-copula, the SJC copula can capture the asymmetry in the upper and lower tail dependence. For a given sample size, the shape of these plots changes considerably as $\tau$ increases.

\subsubsection{Plots for real data}

Figures 4-6 report the chi-plots and K-plots to assess the bivariate dependence between the DM(Euro)/USD and each of the other three exchange rates (JY/USD, SF/USD, BP/USD) for the pre-euro, transition and post-euro sample periods.

Figure 4 shows that there is a significant dependence between the DM(Euro)/USD and JY/USD in the pre-euro and post-euro periods, with clear evidence of tail dependence (joint large appreciations and depreciations) in these periods. In contrast, in the transition period, most of the observations are distributed within the confidence bands, indicating that these exchange rates were largely independent. A comparison of these plots with those obtained from simulated data suggests that both the Student $t$ and the SJC copulas 
are appropriate to model the dependence between the Euro and the Japanese Yen in the pre- and post-euro periods.

The chi-plots for the DM(Euro)/USD-SF/USD reported in Figure 5, on the other hand, show very strong dependence between this exchange rate pair across all three sample periods, as the $\tau$ is close to or above 0.8 . The observations on the right hand side of the plots around $\lambda_{T_{i}}=1$ indicate the presence of significant tail dependence. Overall, it appears that the dependence structure between the DM(Euro) and the SF remained unchanged with the introduction of the Euro. The Student $t$, Gumbel and SJC copulas are all suitable to capture the tail dependence between these two bilateral exchange rates. The chi-plots in Figure 6 exhibit significant dependence between the DM(Euro)/USD and the BP/USD, with an increase in the strength of dependence across the three sample periods. The tail dependence seems to have changed throughout the sample period. From both chi- and K-plots, it appears that the Student t, Clayton and Gumbel copulas would fit the dependence in the first two periods, while the SJC seems suitable for modelling the post-euro period.

\subsection{Copula selection using goodness-of-fit tests}

Following our investigation above, based on visual illustration of dependence, we have implemented the semi-parametric method outlined in section 2.3 to estimate various bivariate copulas for each of the three sample periods, including also time-varying copulas of the kind studied in Patton (2006). We ranked the results for different copulas and for each sample period, according to the goodness of fit test and the AIC, and report the results of the best fitting models in Tables 2-4. These tables also present upper and lower tail dependence parameters $\tau^{U}$ and $\tau^{L}$ (where appropriate) and their corresponding $p$-values for the three pairs of exchange rate returns (Euro/USD-JY/USD, Euro/USDSF/USD, Euro/USD-BP/USD), across the three distinct sample periods (pre-euro, transition, post-euro). As the AIC and goodness of fit test have consistently selected the same copula in all cases, the tables report only the goodness of fit test. We found that the constant SJC copula fits well in most cases.

For the currencies pair Euro/USD-BP/USD, the upper tail dependence (joint large 
depreciations against the dollar) measured by $\tau^{U}$ increased from 0.199 in the pre-euro period to 0.351 in the transition period, to reach a value of 0.472 in the post-euro period. The lower tail dependence (joint large appreciations) measured by $\tau^{L}$, on the other hand, declined in the transition period (from 0.173 to 0.113 ) and showed a marked increase (0.398) in the post-euro period. The upper tail dependence has been higher than the lower tail dependence in all three samples, showing a stronger tendency of the Euro/USD and BP/USD to depreciate together, with the highest difference between the two tail dependence measures during the transition period.

For the pair Euro/USD-JY/USD, in the pre-euro period the dependence in the upper tail (0.383) is higher than in the lower tail (0.305), implying a stronger tendency towards joint large depreciations with respect to the US dollar than towards appreciations. This dependence structure is modified and reversed in the post-euro period, with stronger lower tail dependence (0.345), and slightly weaker upper tail dependence (0.322). Finally, confirming the analysis in the previous section, there is no dependence during the transition period between the Euro and the Japanese Yen. The findings of stronger tail dependence in the pre-euro period and the weakening of the dependence structure between the Euro and the Yen in the first few years of the Euro (transition period) also confirm previous results by Patton (2006) who estimated conditional copulas using a different approach - Inference Function - for the same pair of exchange rate series, but over a shorter period of time (1991-2002). Our sample, extended over five more years, indicates a further change in the dependence structure between the euro and the yen, with a return to a stronger tail dependence, though with a less pronounced asymmetry between lower and upper tails.

A constant normal copula fits best the dependence structure for the pair DM(Euro)/USDSF/USD, capturing the tendency of the Swiss franc to follow the fluctuations of the euro against the US dollar very closely, with an estimate of the correlation coefficient consistently higher than 0.9 . These results are indicative of a high degree of dependence of the Swiss monetary policy relative to the German Bundesbank in the past, and relative to the European Central Bank since 1999, reflecting, on the one hand, the similarities of the economic and policy environment in the euro area and in Switzerland, and, on the other hand, the desire of the Swiss National Bank to ensure the stability of its currency 
against the euro (Swiss National Bank, Monetary Report, 1999). These results are relevant for the question of whether Switzerland should give up its monetary autonomy and join the EU, but also have implications for financial market participants, as investors regard the Swiss franc as a useful means of diversifying the currency composition of their portfolios.

\section{Conclusion}

In this paper we used nonparametric plots, copula models and semiparametric estimation method in order to analyse the dependence structure between the DM(Euro)/USD and each of the following bilaterals: BP/USD, SF/USD and JY/USD, over the period January 1994 to November 2007. These methods have revealed more complicated types of dependence than simple linear association.

As discussed in Patton (2006), while various explanations for the presence of asymmetric dependence between equity returns have been offered in the literature, the possibility of asymmetric dependence between exchange rates has been given much less attention. Following the discussion in Patton (2006), possible asymmetries may arise depending on the relative weight put by central banks on two main objectives: maintaining competitiveness and preserving price stability. A preference for price stability would induce a central bank to intervene in order to match the appreciation of other currencies towards the US dollar. On the other hand, a preference to defend competitiveness would induce central bank intervention to match depreciation when other currencies depreciate against the dollar.

Our findings seem to support the conjecture that the Bank of Japan was more interested in defending price stability in the post-euro period (by intervening to appreciate the yen whenever the euro appreciated against the dollar), while it was more concerned about maintaining export competitiveness before the introduction of the euro (by inducing depreciation of the yen against the dollar to ensure a matching with the depreciation of the euro). Moreover, while there is significant evidence that the pair Euro/USD-BP/USD 
have been linked to one another with increasing strength over the post euro period and with stronger dependence during depreciations than appreciations, for the pair Euro/USD-SF/USD the evidence of comovements is much more compelling, a result which can be of interest when addressing issues related to the degree of independence of Swiss monetary policy relative to the ECB and to the Bundesbank in the past.

Understanding the nature of the conditional dependence between foreign exchange rates and changes in its structure over time is of relevance for financial decisions related to currency portfolio management and international portfolio diversification exposed to the risk of exchange rate fluctuations.

\section{References}

Ang, A. and Bekaert, G. (2002). International Asset Allocation With Regime Shifts. The Review of Financial Studies 15, 1137-1187.

Ang, A. and Chen, J. (2002). Asymmetric Correlations of Equity Portfolios. Journal of Financial Economics 63, 443-94.

Bank for International Settlements (2007). Triennal Central Bank Survey - Foreign exchange and derivatives market activity in 2007. (December).

Bouyé, E. and Salmon, M. (2002). Dynamic Copula Quantile Regression and Tail Area Dynamic Dependence in Forex Markets. Manuscript, Financial Econometrics Research Centre, Warwick Business School, UK.

Breymann, W., Dias, A. and Embrechts, P. (2003). Dependent structures for multivariate high-frequency data in finance. Quantitative Finance 3, 1-14

Campbell, R., Forbes, C., Koedijk, K. and Kofman, P. (2008). Journal of Empirical Finance, 15, 2, 287-309

Clayton,D. G. (1978). A Model for Association in Bivariate Life Tables and Its Application in Epidemiological Studies of Family Tendency in Chronic Disease Incidence. Biometrika 65, 141-51.

Copeland, L. (2008). Exchange Rates and International Finance, $5^{\text {th }}$ Edition, Pearson Education Limited. 
Dias, A. and Embrechts, P. (2007). Testing for structural changes in exchange rates dependence beyond linear correlation. European Journal of Finance (forthcoming).

Embrechts, P., McNeil, A. and Straumann, D. (2002). Correlation and Dependence Properties in Risk Management: Properties and Pitfalls, in M. Dempster, ed., Risk Management: Value at Risk and Beyond, Cambridge University Press.

Fisher, N. and Switzer, P. (1985). Chi-Plots forAssessing Dependence. Biometrika 72, 253-265

Fisher, N. and Switzer, P. (2001). Graphical Assessment of Dependence: is a Picture Worth 100 Tests? The American Statistician 55, 233-239.

Genest, C. and Boies, J. (2003). Detecting Dependence with Kendall Plots. The American Statistician 57(4), 275-284.

Genest, C., Glhoudi, K. and Rivest, L. P. (1995). A semiparametric Estimation Procedure of Dependence Parameters in Multivariate Families of Distributions, Biometrika $82(3), 543-552$

Granger, C. and Silvapulle, P. (2001). Large Returns, Conditional Correlation and Portfolio Diversification: a Value-at-Risk Approach. Quantitative Finance 1, 542551.

Gumbel, E.J. (1960). Bivariate exponential distributions. J. Am. Stat. Assoc. 55, 698-707.

Hurd, M., Salmon, M. and Schleicher, C. (2005). Using copulas to construct bivariate foreign exchange distributions with an application to the sterling exchange rate index. Financial Econometrics Research Centre, Warwick Business School, Working Paper, WP05-20.

Joe, H. (1997). Multivariate Models and Dependence Concepts. Boca Raton London New York Washington, D.C.: Chapman and Hall/CRC.

Jondeau, E. and Rockinger, M. (2006). The copula-GARCH model of conditional dependencies: an international stock market application, Journal of International Money and Finance, 25(5), 827-853.

Kendall, M. G. (1938). A new measure of rank correlation. Biometrika 30, 81-93.

Kim, G., Silvapulle, M. J. and Silvapulle, P. (2007a). Comparison of Semiparametric and Parametric Methods for Estimating Copulas. Computational Statistics and Data Analysis 51, 2836-2850. 
Kim, G., Silvapulle, M. J. and Silvapulle, P. (2007b). Semiparametric Estimation of the Error Distribution in Multivariate Regression Using Copulas. Australian and New Zealand Journal of Statistics 49(3), 321-336.

Kim, G., Silvapulle, M. J. and Silvapulle, P. (2008). Estimating the error distribution in multivariate heteroscedastic time-series models. Journal of Statistical Planning and Inference 138, 1442-1458.

Login, F. and Solnik B. (2001). Extreme Correlation of International Equity Markets. Journal of Finance 56, 649-676.

Nelsen, R. B. (2006). An Introduction to Copulas. New York: Springer Series in Statistics.

Patton, A. (2006). Modelling Asymmetric Exchange Rate Dependence. International Economic Review 47(2), 527-556.

Patton, A.(2007). Copula-based models for financial time series. In Andersen, T.G., Davis, R.A., Kreiss, J. P., Mikosch T. (eds.) Handbook of Financial Time Series, Springer Verlag, forthcoming.

Rodriguez, J. C. (2007). Measuring Financial Contagion: A Copula Approach. Journal of Empirical Finance 14, 401-423.

Sarno, L. and Taylor, M. (2002). The economics of exchange rates. Cambridge University Press.

Sklar, A. (1959). Fonctions de répartition à $\mathrm{n}$ dimentions et leurs marges. Pub. Inst. Statist. Univ. Paris 8, 229-231.

Sun, W., Rachev, S., Stoyan, V. and Fabozzi, F. (2008). Multivariate Skewed Student's t Copula in the Analysis of Nonlinear and Asymmetric Dependence in the German Equity Market. Studies in Nonlinear Dynamics and Econometrics 12 (2), article 3. 
Table 1: Descriptive statistics of the exchange rate returns series

\begin{tabular}{|c|c|c|c|c|}
\hline & $\mathrm{DM}($ Euro)/USD & JY/USD & SF/USD & BP/USD \\
\hline & \multicolumn{4}{|c|}{ Pre-euro period (January 1994 - December 1998) } \\
\hline Mean & -0.003 & 0.001 & -0.006 & -0.010 \\
\hline Std. deviation & 0.591 & 0.790 & 0.687 & 0.525 \\
\hline Skewness & -0.254 & -0.841 & -0.381 & -0.102 \\
\hline Kurtosis & 5.064 & 9.770 & 5.737 & 9.380 \\
\hline Jarque Bera test & $241.24^{*}$ & $2597.51^{*}$ & $429.02^{*}$ & $2042.61^{*}$ \\
\hline ARCH LM test & $77.06^{*}$ & $118.54^{*}$ & $91.56^{*}$ & $118.25^{*}$ \\
\hline Pearson's $\rho$ & & 0.536 & 0.932 & 0.336 \\
\hline Kendall's $\tau$ & & 0.367 & 0.751 & 0.227 \\
\hline \multirow[t]{2}{*}{ Number of obs. } & & 1281 & 1276 & 1203 \\
\hline & \multicolumn{4}{|c|}{ Transition period (January 1999 - February 2002) } \\
\hline Mean & 0.038 & 0.021 & 0.026 & 0.019 \\
\hline Std. deviation & 0.656 & 0.666 & 0.659 & 0.509 \\
\hline Skewness & -0.499 & -0.225 & -0.494 & -0.119 \\
\hline Kurtosis & 4.315 & 4.264 & 4.341 & 3.563 \\
\hline Jarque Bera test & $92.30^{*}$ & $61.03^{*}$ & $93.60^{*}$ & $12.20^{*}$ \\
\hline ARCH LM test & 8.60 & $31.16^{*}$ & 6.20 & 13.71 \\
\hline Pearson's $\rho$ & & 0.118 & 0.938 & 0.425 \\
\hline Kendall's $\tau$ & & 0.084 & 0.802 & 0.289 \\
\hline \multirow[t]{2}{*}{ Number of obs. } & & 813 & 818 & 783 \\
\hline & \multicolumn{4}{|c|}{ Post-euro period (March 2002 - November 2007) } \\
\hline Mean & -0.035 & -0.010 & -0.027 & -0.027 \\
\hline Std. deviation & 0.543 & 0.572 & 0.613 & 0.513 \\
\hline Skewness & 0.042 & -0.381 & -0.018 & 0.103 \\
\hline Kurtosis & 3.663 & 4.759 & 3.540 & 3.600 \\
\hline Jarque Bera test & $27.09^{*}$ & $223.17^{*}$ & $17.74^{*}$ & $24.38^{*}$ \\
\hline ARCH LM test & $60.91^{*}$ & 8.76 & $40.01^{*}$ & $41.57^{*}$ \\
\hline Pearson's $\rho$ & & 0.528 & 0.947 & 0.633 \\
\hline Kendall's $\tau$ & & 0.357 & 0.792 & 0.438 \\
\hline Number of obs. & & 1457 & 1447 & 1436 \\
\hline
\end{tabular}

Note: An asterisk $(*)$ indicates that the null hypothesis is rejected at the $5 \%$ significance level. 
Table 2. Estimated copula parameters for the DM(Euro)/USD and BP/USD

\begin{tabular}{|c|c|c|c|}
\hline Parameter & Pre-euro & Transition & Post-euro \\
\hline & \multicolumn{3}{|c|}{ Constant normal copula } \\
\hline$\rho$ & 0.351 & 0.423 & 0.636 \\
\hline standard error & $(0.022)$ & $(0.026)$ & $(0.013)$ \\
\hline Goodness of fit test* & 107.973 & 57.422 & 45.964 \\
\hline \multirow[t]{2}{*}{ p-value } & $(0.253)$ & $(0.191)$ & $(0.597)$ \\
\hline & & Constant $S$ & \\
\hline$\tau^{\mathrm{U}}$ & 0.199 & 0.351 & 0.472 \\
\hline standard error & $(0.039)$ & $(0.041)$ & $(0.021)$ \\
\hline$\tau^{\mathrm{L}}$ & 0.173 & 0.113 & 0.398 \\
\hline standard error & $(0.037)$ & $(0.046)$ & $(0.042)$ \\
\hline Goodness of fit test* & 109.968 & 53.079 & 57.454 \\
\hline p-value & $(0.212)$ & $(0.320)$ & $(0.191)$ \\
\hline
\end{tabular}

Table 3. Estimated copula parameters for the DM(Euro)/USD and JY/USD

\begin{tabular}{|c|c|c|c|}
\hline Parameter & Pre-euro & Transition & Post-euro \\
\hline & \multicolumn{3}{|c|}{ Constant normal copula } \\
\hline$\rho$ & 0.543 & 0.104 & 0.529 \\
\hline standard error & $(0.017)$ & $(0.035)$ & $(0.016)$ \\
\hline Goodness of fit test* & 81.295 & 42.412 & 54.428 \\
\hline \multirow[t]{2}{*}{ p-value } & $(0.902)$ & $(0.736)$ & $(0.059)$ \\
\hline & \multicolumn{3}{|c|}{ Constant SJC copula } \\
\hline$\tau^{\mathrm{U}}$ & 0.383 & 0.059 & 0.322 \\
\hline standard error & $(0.041)$ & $(0.050)$ & $(0.039)$ \\
\hline$\tau^{\mathrm{L}}$ & 0.305 & 0.000 & 0.345 \\
\hline standard error & $(0.040)$ & $(-)$ & $(0.044)$ \\
\hline Goodness of fit test* & 100.811 & 41.797 & 39.465 \\
\hline p-value & $(0.430)$ & $(0.758)$ & $(0.833)$ \\
\hline
\end{tabular}

Table 4. Estimated copula parameters for the DM(Euro)/USD and SF/USD

\begin{tabular}{|c|c|c|c|}
\hline Parameter & Pre-euro & Transition & Post-euro \\
\hline & \multicolumn{3}{|c|}{ Constant normal copula } \\
\hline$\rho$ & 0.927 & 0.938 & 0.945 \\
\hline standard error & $(0.003)$ & $(0.001)$ & $(0.002)$ \\
\hline Goodness of fit test* & 97.354 & 74.910 & 47.229 \\
\hline $\mathrm{p}$-value & $(0.528)$ & $(0.736)$ & $(0.545)$ \\
\hline
\end{tabular}

*This is the goodness of fit test proposed by Breymann et al. (2003). 
Figure 1. Exchange rates against the US dollar, January 1994-November 2007 (4 January 1999=100).
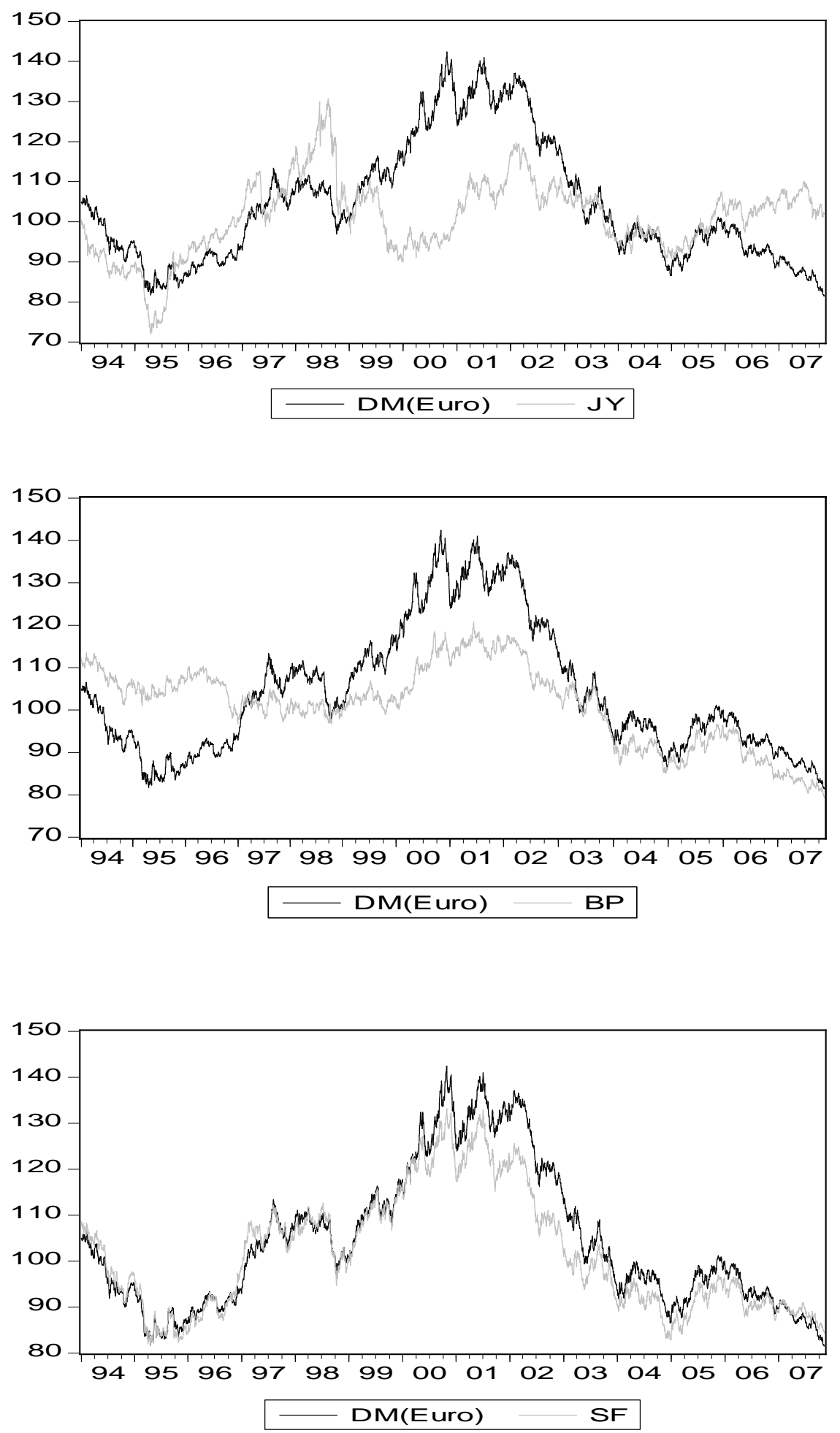
Figure 2. Chi and Kendall plots for the theoretical copulas $(\tau=0.792$, sample size $=1250)$.
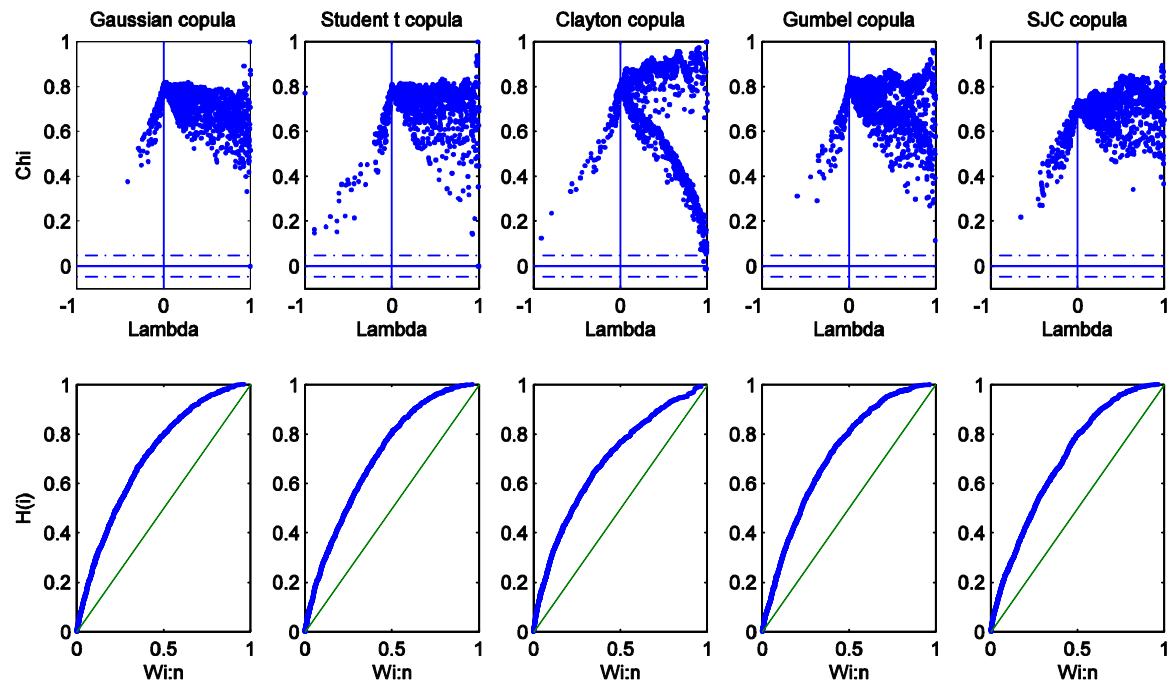

Figure 3. Chi and Kendall plots for the theoretical copulas $(\tau=0.438$, sample size $=1450)$.
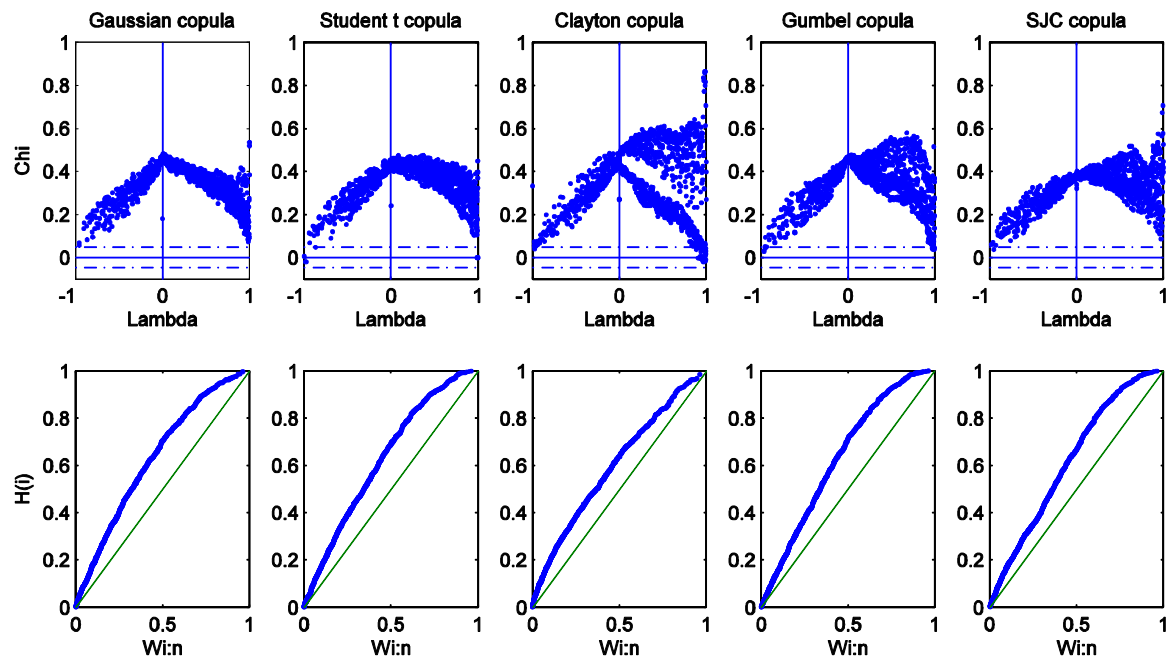
Figure 4. Chi and Kendall plots for the DM(Euro)/USD and JY/USD exchange rates.
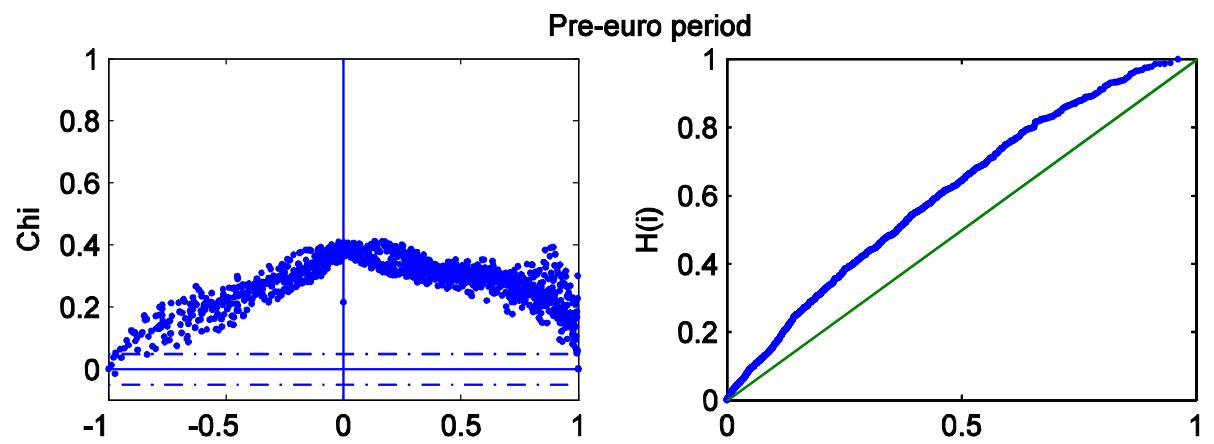

Transition period
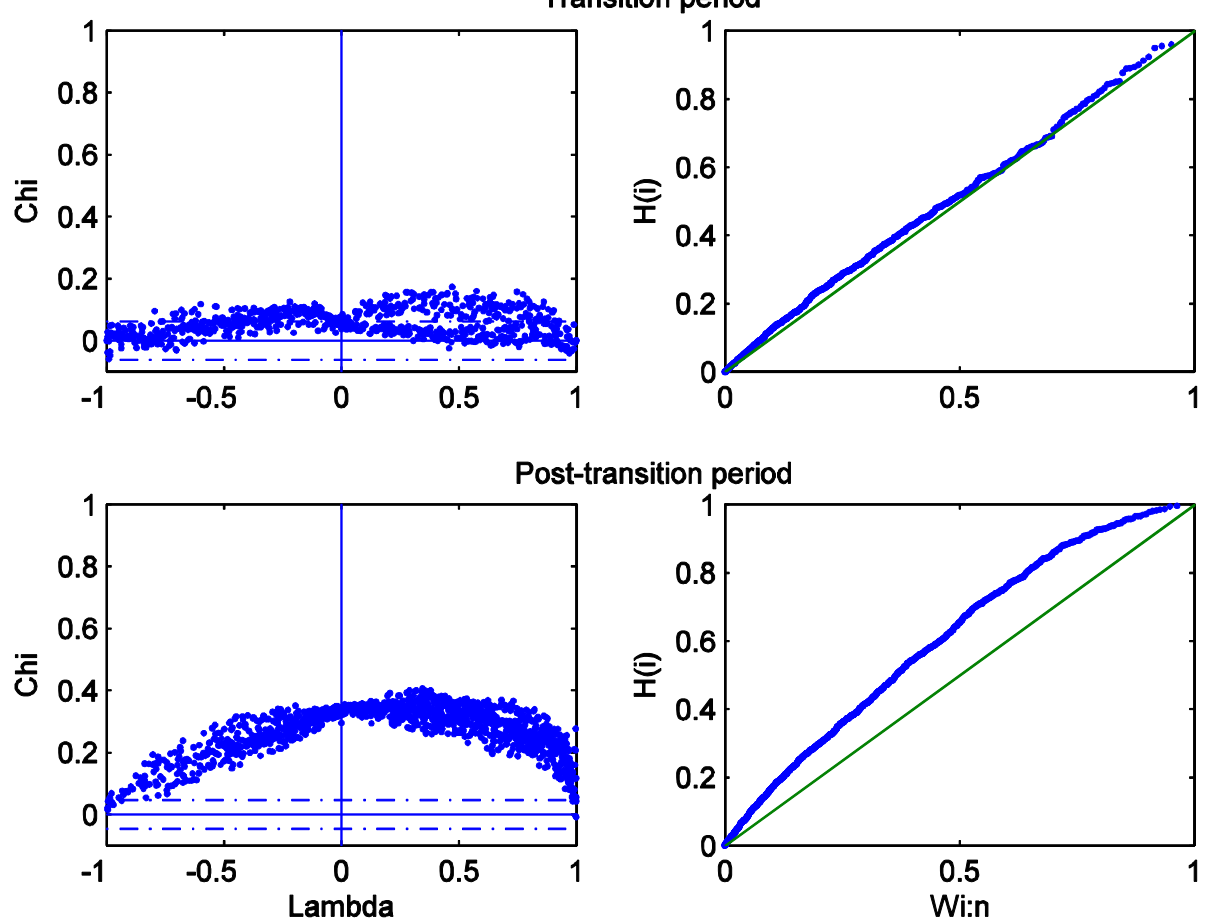
Figure 5. Chi and Kendall plots for the DM(Euro)/USD and SF/USD exchange rates.
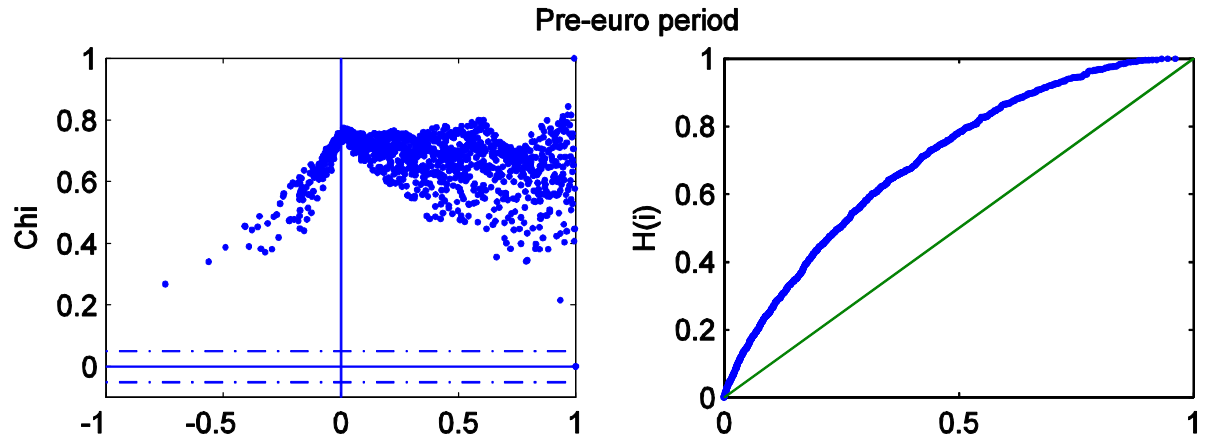

Transition period
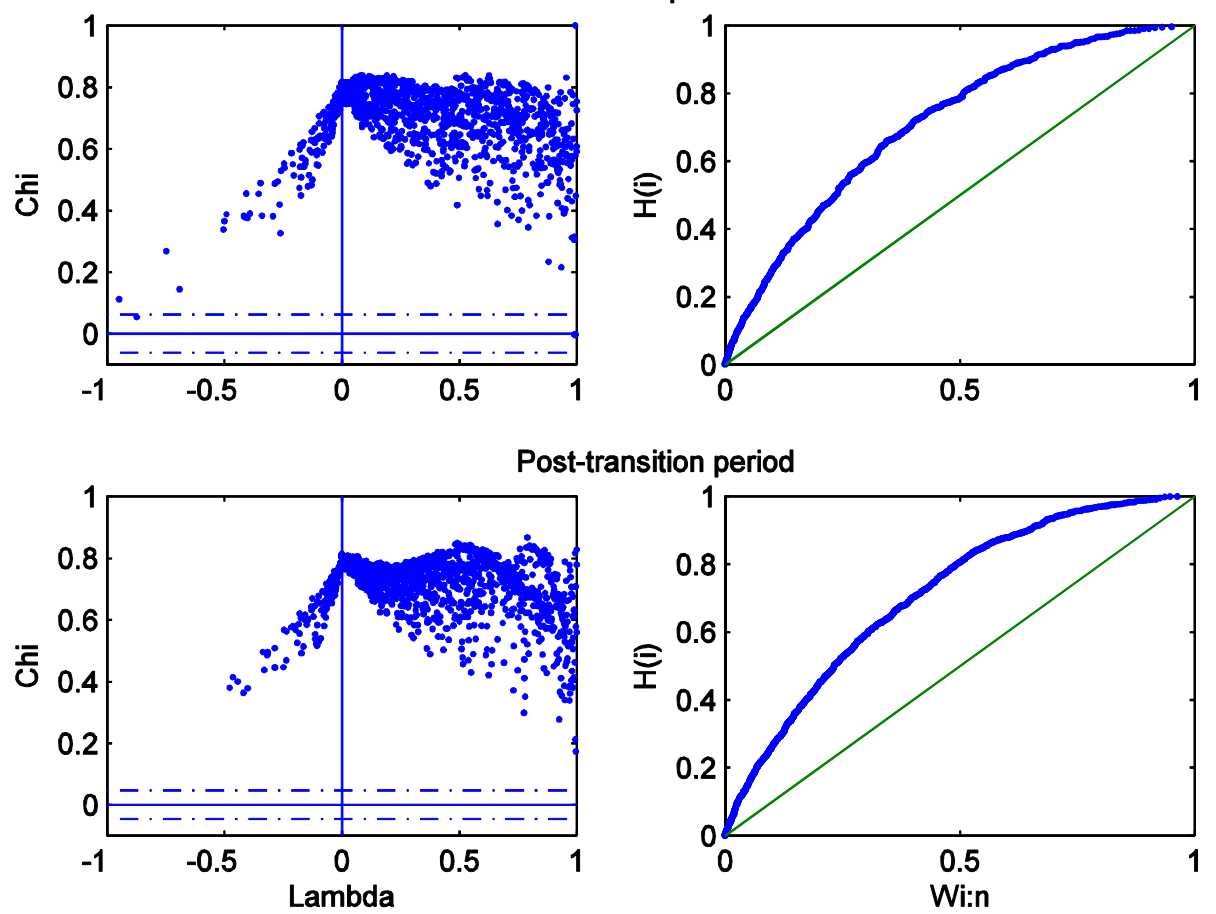
Figure 6. Chi and Kendall plots for the DM(Euro)/USD and BP/USD exchange rates.
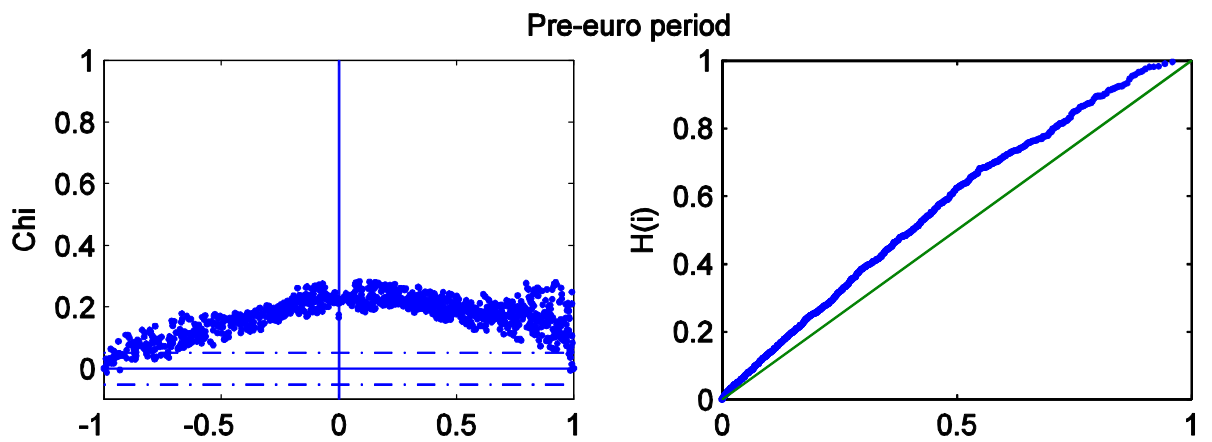

Transition period
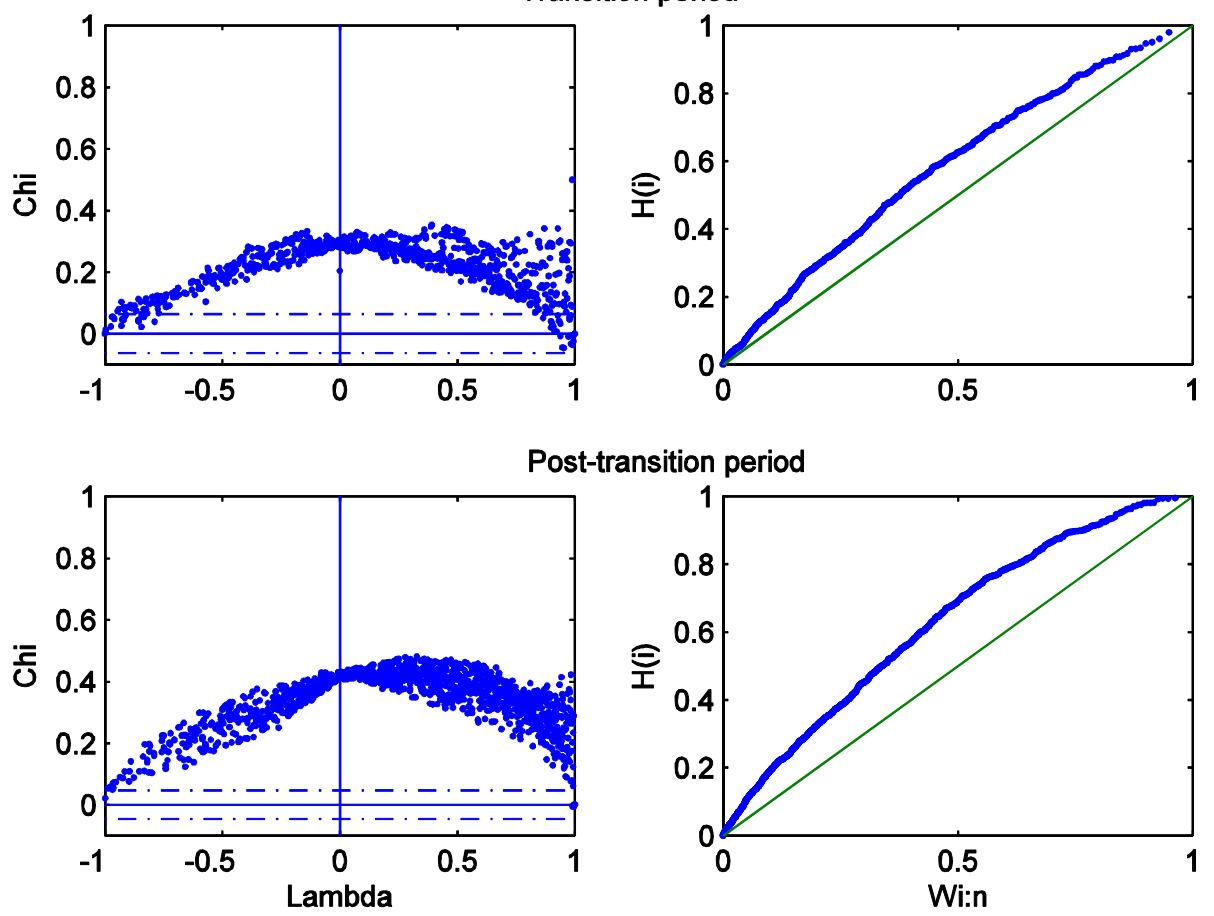